Carestream, UCB, Abbvie, Pfizer, Astra Zeneka, Roche, Siemens, Image Analysis Group, Jonathan Vela: None declared, Soeren Skou: None declared, Mikkel Eld: None declared, Nasir Musa Al-Mashkur: None declared, Robert Riis Consultant for: Image analysis Group, Ole Simonsen: None declared

DOI: 10.1136/annrheumdis-2019-eular.5695

\section{AB1126 ULTRASOUND CHANGES IN PATIENTS WITH CALCIFICATIONS IN THE SUPERSPINATUS TENDON BEFORE AND AFTER THE DEVELOPMENT OF A SUBACROMIAL PAINFUL SYNDROME}

Carlos Guillén-Astete, Andrea Briones-Figueroa. Ramon y Cajal University Hospital, Madrid, Spain

Background: The relationship between subacromial syndrome (SS) and the presence of calcifications in the thickness of the superspinatus tendon is complex. It is undeniable that the presence of a solid body hinders the movement of the rotator cuff through the subacromial space and to some extent contributes to the trapping that conditions pain and functional limitation, but, on the other hand, calcifications in the thickness of the supraspinatus tendon are incidental findings that have no clinical significance or whose presence precedes the painful syndrome in months or years. We understand that in a SS related to calcification, it contributes as an irreducible agent that conditions an inflammatory response of the tendon when it attempts to cross the subacromial space, however, there are no studies available that explain this relationship from a sonoanatomic perspective.

Objectives: To determine the extent to which the disposition and characteristics of a calcification of the supraspinatus tendon, or of the tendon itself, are modified when an SS is triggered.

Methods: A quasi-experimental, "before-after" study was conducted with individuals who presented a SS and in whom incidental calcification in the TSE had been previously diagnosed in an ultrasound study. Demographic data and static images from the ultrasound studies of each patient were compared. The explanatory variable was time (before-after) and the response variables in image were: (1) Increased tendon thickness measured at $30 \mathrm{~mm}$ from the deep edge of the acromion (GROSORB), (2) Increased tendon thickness measured at the height of the middle of the longitudinal axis of the calcification (GROSORC), (3) Longitudinal axis of the calcification (LONGC), (4) Thickness or height of the calcification, when this was measurable (ALTC), and (5) Distance between the calcification and the deep end of the acromion (DISTC). In addition, two indices were determined: (a) Increased tendon thickness in the calcification zone (GROSORC/GROSORB) and (b) proportion of tendon thickness occupied by calcification (ALTC/GROSORC). The measurements were carried out using the software of the equipment in which the original and subsequent study was made (GE Logiq e; Toshiba Nemio $X G)$

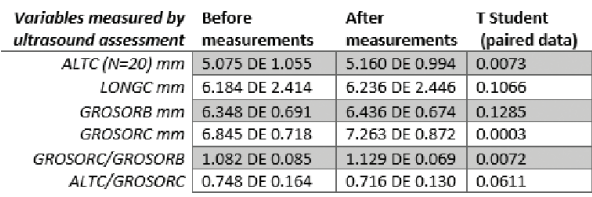

Results: The "before" ultrasounds were performed between 2012 and 2016 and the "after" ultrasounds between 2015 and 2018. The time fashion between the two ultrasounds for each patient was 2 years. Thirtythree subjects were included, with an average age of 47.5 SD 7.6 years. Proportion of women $66.6 \%$. Proportion of dominant shoulders $(51.1 \%)$. As shown in table 1, the most significant changes were the thickness of the tendon measured at the height of the middle of the longitudinal axis of calcification and the increase of this measure with respect to the thickness measured at $30 \mathrm{~mm}$ from the deepest region of acromion (GROSORC/GROSORB). Although the calcification height also showed a statistically significant increase, it could only be measeured in 20 of the 33 subjects.

In the correlation tests, the GROSORC/GROSORB index increase correlated strongly with the DISTC (Coef. Pearson 0.686, P<0.01) and with the longitudinal diameter of the calcification (LONGC) (Coef. Pearson $0.797, P<0.01)$
Conclusion: The most important factor that explains the development of an SS associated with calcification is the increase in tendon thickness at the height of the calcification itself and, at the same time, this measure increases when the calcification is more lateralized and the more elongated its longitudinal diameter. These modifications could have implications when deciding the therapeutic management of these processes.

Disclosure of Interests: None declared

DOI: 10.1136/annrheumdis-2019-eular.5918

\section{AB1127 ULTRASOUND INTER-READER RELIABILITY OF INFLAMMATORY FINDINGS IN PATIENTS WITH POLYARTHRITIS}

Luisa Brites $^{1}$, Mariana Santiago ${ }^{1,2}$, João Rovisco ${ }^{1,2}$, João Dinis de Freitas ${ }^{1}$, José Antonio P. Da Silva ${ }^{1}, 2$, Inês Silva ${ }^{3}$, Filipa Teixeira ${ }^{4}$, Daniela Faria ${ }^{4}$, Cândida G. Silva ${ }^{5,6}$, Sandra Falcão ${ }^{3,7} .{ }^{1}$ Centro Hospitalar e Universitário de Coimbra, Coimbra, Portugal; ${ }^{2}$ Faculdade de Medicina de Coimbra, Coimbra, Portugal; ${ }^{3}$ Hospital Egas Moniz, Centro Hospitalar de Lisboa Ocidental, Lisbon, Portugal; ${ }^{4}$ Unidade Local de Saúde do Alto Minho, Serviço de Reumatologia, Ponte de Lima, Portugal; ${ }^{5}$ Higher School of Health Sciences, Polytechnic Institute of Leiria, Leiria, Portugal; ${ }^{6}$ Centro de Quimica da Universidade de Coimbra, Coimbra, Portugal;

${ }^{7}$ Faculdade de Ciências Médicas da Universidade Nova de Lisboa, Lisbon, Portugal

Background: Ultrasonography is an imaging technique that allows rheumatologists to visualise structural and inflammatory changes within a joint. Objectives: The objective of this study was to assess the inter-reader reliability of interpretation of inflammatory and destructive changes in a wide range of joints in patients with polyarthritis.

Methods: This study was divided in two parts: 1) consensus process and 2) reliability exercise. For the first part, a written questionnaire was sent by email to 6 sonographers from 3 portuguese hospitals with the highest level of competence (EULAR competency assessment level 2). The questionnaire included 17 questions divided in two groups: 1) elementary components in B-mode and Doppler assessment (effusion, synovial hypertrophy $(\mathrm{SH})$, power Doppler ( $\mathrm{PD})$, erosions and synovitis definition) and 2) approach at the joint level (the definition of which plan and recess will be assessed in each joint). The participants were asked to rate their level of agreement/disagreement for each statement using a 1-5 Likert scale ( $1=$ strongly disagree to 5 -strongly agree). For the reliability exercise, video clips of US examinations of 40 joints (wrist, metacarpophalangeal (MCP) from 1 to 5 , proximal interphalangeal (PIP) from 1 to 5 , knee, tibiotarsal (TT) and metatarsophalangeal (MTP) joints from 1 to 5, elbow and shoulder) from each of 15 patients were collected (showing a multiplanar bilateral ultrasound approach). Each joint in each video was scored by individual ultrasonographers for the presence/absence of elementary components: effusion (Yes/No), SH (No/Grade 1 to 3), PD (No/ Grade 1 to 3 ) and erosions (Yes/No). Inter-reader agreement analysis was assessed through Fleiss' kappa coefficient and classified according to Landis and Koch[8]: $\kappa$ values 0 were considered poor, $0-0.20$ slight, 0.21-0.40 fair, 0.41-0.60 moderate, 0.61-0.80 good and 0.81-1.00 excellent. Statistical significance was defined as $\mathrm{p}<0.05$. Statistical analysis was performed using STATA V.14.

Results: Thirty seven joints of the 600 joints were excluded due to dislocation of the joint or presence of objects (rings/catheters) and the videos of a total of 563 joints were analysed by the 6 ultrasound experts. Interreader agreement was superior for TT joints and inferior for wrist; the identification of the erosions had the better agreement in the elementary components (Table 1)

Table 1. Inter-observer agreement for each elementary component and for anatomical region

\begin{tabular}{|c|c|c|c|c|c|}
\hline $\begin{array}{l}\text { Elementary } \\
\text { component }\end{array}$ & $\kappa$ & Joint & $\kappa$ & Joint & $\kappa$ \\
\hline Effusion & 0.6044 & Wrist & 0.6767 & Shoulder & 0.7271 \\
\hline $\begin{array}{l}\text { Synovial } \\
\text { hypertrophy }\end{array}$ & 0.6291 & MCP & 0.6866 & Knee & 0.7192 \\
\hline Power doppler & 0.7195 & PIP & 0.7107 & $\mathrm{TT}$ & 0.8043 \\
\hline Erosions & 0.7314 & Elbow & 0.7291 & MTP & 0.7040 \\
\hline
\end{tabular}

interphalangeal joints, TT-Tibiotarsal joint. All $p<0.001$

Conclusion: The reliability of interpretation of inflammatory and destructive changes using video clips was in general good to excellent and it was better for erosions and tibiotarsal joint (regarding elementary component and anatomical region, respectively).

Disclosure of Interests: None declared 